\title{
Alcohol withdrawal
}

\author{
Ashish Sarangi MD
}

\begin{abstract}
Objective: This case review discusses the current recommended protocol for the diagnosis and treatment of delirium tremens using a review of a patient managed both in an inpatient psychiatry unit and in an intensive care unit. This review and case study are intended to help guide patient care with co-morbid conditions confounding diagnosis and in facilities lacking sophisticated monitoring equipment.

Data Sources: PubMed was searched using keywords and phrases, including delirium tremens, alcohol withdrawal, chronic alcoholism, CIWA, alcohol effect on CNS, treatment of delirium tremens, and treatment of alcohol withdrawal.

Study Selection: The case discussed provided the basis for this report based on its complexity due to comorbid conditions and the initial subacute presentation.

Data Extraction: Data were gathered from charting notes written concurrently with patient management. Vital signs and laboratory values were regularly measured, and healthcare faculty documented each clinical encounter with findings and updates to treatment.

Results: Delirium tremens was identified before progression to seizures and severe autonomic instability. Appropriate treatment and transfer to an intensive care unit were secured once a significant index of suspicion was reached.

Conclusions: Close and frequent patient evaluation despite the lack of continuous monitoring technology allowed detection of decompensation as it began insidiously. The gradual development of cognitive symptoms and the presence of abnormal laboratory results helped identify his deterioration. The current guidelines for the treatment of delirium tremens provide the basis for the management of this highly dangerous disorder.
\end{abstract}

Keywords: Delirium tremens, alcohol withdrawal, biomarkers

\section{INTRODUCTION}

Despite the negative health effects of frequent alcohol intoxication, significant numbers of men $(20 \%)$ and women $(10 \%)$ in most Western societies will develop an alcohol-use disorder during their lifetime. ${ }^{1}$ Once a pattern of use and dependency emerges, managing alcohol withdrawal syndrome (AWS) when substance use is ended or interrupted becomes vital. The rate of progression to severe withdrawal can vary, and the

Corresponding author: Ashish Sarangi

Contact Information: Ashish.Sarangi@ttuhsc.edu

DOI: 10.12746/swrccc.v8i35.717 constellation of associated symptoms, while fairly welldefined, can be missed. In addition, patients hospitalized for reasons other than alcohol use may not report their usage, leading to unexpected withdrawal when unable to maintain alcohol intake. Nearly $20 \%$ of the population reports some form of alcohol abuse, but studies indicate that $40 \%$ of young adult patients with AWS were not identified and $60 \%$ of elderly patients were missed. ${ }^{2}$

Typically, patients with AWS present with autonomic excitation and agitation. ${ }^{3}$ However, some patients insidiously progress to a severe form known as delirium tremens (DTs) over time, manifesting as delirium along with a mixture of hallucinations, seizures, autonomic instability, mydriasis, nausea and vomiting, diaphoresis, anxiety, agitation, and tremor. ${ }^{1,2}$ The diagnosis of 
DTs requires a detailed history with close monitoring of cognitive function and vital signs. Although tests like the revised Clinical Institute Withdrawal Assessment for Alcohol (CIWA-Ar) are widely used as indicators of withdrawal severity and in protocols for management, it is likely that centers lacking these resources would have trouble managing these patients. ${ }^{4}$

This report showcases the insidious onset of DT in a psychiatric inpatient setting and suggests that biomarkers that could improve the detection of impending DTs.

\section{CASE}

This patient, a middle-aged Hispanic male, began drinking at the age of 15 progressing to $475 \mathrm{~mL}$ (1 pint) of vodka daily. Three years ago, he attempted to quit but was unsuccessful. Two months ago, he received a DWI citation. After experiencing severe hangover with intense vomiting, he again resolved to quit. He visited his primary care physician and was told that he should not attempt to quit without medical help. In addition to alcohol use, he had hypertension, treated with labetalol. He had no other significant psychiatric, medical, or surgical history and had no known drug allergies.

The patient presented to the emergency department (ED) at 11:00 for alcohol detoxification, three days after his last drink. He complained of vomiting over the past two days, totaling 40-50 times. He also reported chills, trembling, and insomnia. Upon physical examination, he had dry mucous membranes, and his blood pressure was $133 / 91 \mathrm{mmHg}$. He received $1 \mathrm{~L}$ of saline and $40 \mathrm{mEq}$ of potassium chloride $(\mathrm{KCl})$ for volume expansion and hypokalemia. He refused ondansetron and denied abdominal pain. Liver enzymes (AST and ALT) were elevated at $126 \mathrm{IU} / \mathrm{L}$ and $140 \mathrm{IU} / \mathrm{L}$, respectively; alkaline phosphatase was within normal limits at $25 \mathrm{IU} / \mathrm{L}$. His hemoglobin was within normal limits at $13.2 \mathrm{gm} / \mathrm{dL}$, and he had a platelet count of $82 \mathrm{k} / \mu \mathrm{L}$.

Nine hours after arrival, a consult was called for admission to the inpatient psychiatric hospital since he was stable. He denied changes in attention, vision, energy, anxiety, hallucinations, and any history of seizures. Mild tremors in his hands upon extension were noted. No other psychiatric findings were present. His electrocardiogram showed normal sinus rhythm, nonspecific ST abnormality, and a prolonged QT interval.

The patient was transferred to inpatient psychiatric facilities and interviewed the following morning at 10:00. Hypokalemia was still present at 04:00 on his first hospital day and was corrected with intravenous (IV) $\mathrm{KCl} 50 \mathrm{mEq}$ at 10:00. ALT and AST levels remained elevated at $122 \mathrm{IU} / \mathrm{L}$ and $104 \mathrm{IU} / \mathrm{L}$, and he had hyperbilirubinemia at $2.2 \mathrm{mg} / \mathrm{dL}$, hyperammonemia at $52 \mathrm{mmol} / \mathrm{L}$, and a prolonged QT interval (494 ms). $\mathrm{He}$ reported auditory hallucinations overnight but denied tactile or visual components at that time and current hallucinations. Tremors were evident in both upper extremities. Testing with the Montreal Cognitive Assessment showed clear deficits in serial 7s subtraction and inability to spell "world" backwards. He recalled 2 of 3 provided words and provided an incorrect drawing of a clock. He remained stable on the unit until 15:30 on hospital day 1 .

At 15:30, the patient became restless and confused, pacing the halls and experiencing visual hallucinations. He was disoriented to time and place and poorly aware of his deficits. Another Montreal Cognitive Assessment was attempted, and he failed to complete any section to satisfaction. The psychiatric team suspected impending DTs and transferred him to the ED for severe alcohol withdrawal at 18:00 on hospital day 1 .

The total duration of hospitalization was 4 days, and the patient was monitored with CIWA-Ar protocol by the nursing staff. On the second hospital day, he was moved to the medical intensive care unit due to worsening symptoms. At intake, he exhibited tremors, progressive confusion, agitation, reduced awareness, auditory and visual hallucinations, and rapidly variable cognitive ability. These are hallmark symptoms of DTs, indicating a high risk for seizures. He denied chills, diaphoresis, nausea, headache, or dizziness. He remained tachycardic with an elevated BP. The patient was oriented during interview but had poor insight and was unaware of why he was still hospitalized. He believed he was improving and going to be discharged despite being previously informed of his deteriorating condition when obtaining permission to transfer. AST 
Table 1. Vital Signs During Hospitalization

\begin{tabular}{|c|c|c|c|c|}
\hline Time & $\begin{array}{c}\text { Temperature } \\
{ }^{\circ} \mathbf{F} \\
\end{array}$ & $\begin{array}{c}\text { Pulse } \\
\text { Beats/minute }\end{array}$ & $\begin{array}{c}\text { Respiratory Rate } \\
\text { Breaths/minute }\end{array}$ & $\begin{array}{c}\text { Blood Pressure } \\
\text { mmHg }\end{array}$ \\
\hline $\begin{array}{l}\text { ED } \\
11: 33\end{array}$ & 97.2 & 121 & 18 & $133 / 91$ \\
\hline $15: 31$ & 97.2 & 89 & 18 & $140 / 83$ \\
\hline $\begin{array}{l}\text { HD1 } \\
8: 37\end{array}$ & - & 127 & - & $130 / 92$ \\
\hline $15: 15$ & 98.3 & 111 & 18 & $137 / 87$ \\
\hline $23: 27$ & 97 & 112 & 26 & $153 / 120$ \\
\hline $\begin{array}{l}\text { HD2 } \\
05: 15\end{array}$ & - & 77 & 14 & $132 / 102$ \\
\hline 16:00 & - & 88 & 22 & $133 / 94$ \\
\hline $\begin{array}{l}\text { HD3 } \\
4: 00\end{array}$ & 99.5 & 90 & 80 & $127 / 90$ \\
\hline $14: 00$ & - & 91 & 17 & $146 / 96$ \\
\hline $22: 18$ & 98.4 & 103 & 20 & $128 / 76$ \\
\hline $\begin{array}{l}\text { HD4 } \\
00: 09\end{array}$ & 98.7 & 112 & 20 & $144 / 94$ \\
\hline $9: 28$ & 98.4 & 103 & 16 & $145 / 92$ \\
\hline
\end{tabular}

and ALT levels increased on hospital day 2 to $132 \mathrm{IU} / \mathrm{L}$ and $122 \mathrm{I} \mathrm{U} / \mathrm{L}$, respectively. Thrombocytopenia persisted on days 2 and 3 .

On hospital day 3 the patient was recovering and denied lingering symptoms. On hospital day 4 , he was no longer experiencing withdrawal symptoms except mild tremor and requested discharge. He was diagnosed with alcoholic cirrhosis of the liver, alcohol use disorder, hypertension, and thrombocytopenia. His DTs had resolved. He was discharged with prescriptions for lorazepam as needed for agitation and continued labetalol and was instructed to remain on a cardiac diet and follow up with outpatient rehabilitation. His clinical course, including vital signs, medication, and laboratory tests, is summarized in Tables 1, 2, and 3.

\section{Discussion}

To treat people with alcoholism adequately, clinicians need tools that can properly assess not only the extent of the patients' recent and past drinking activities but also any relevant family history of drinking problems (i.e., genetic predispositions to alcohol abuse and alcoholism). A good case history provides a start, but more objective measures also are important. Biochemical substances in the body that indicate the presence or progression of a condition or any genetic predisposition toward it are called biomarkers. There are two kinds: state markers and trait markers. State markers are biochemical measures that tell clinicians something about people's recent drinking patterns, including whether they have a history of heavy drinking and whether they have had a recent binge or even just a few drinks. Trait markers are biochemical markers that reveal something about a person's inherited risk of abusing alcohol. A good biomarker, whether state or trait, should be sensitive, i.e., accurate for most if not all drinkers not just a subset, and specific, i.e., linked to alcohol use but not other clinical problems. The test used to measure the biomarker also should be precise and accurate. 
Table 2. Medication Totals Per Day

\begin{tabular}{|l|c|c|c|c|c|}
\hline Medication & ED & HD1 & HD2 & HD3 & HD4 \\
\hline Potassium Chloride & $40 \mathrm{mEq}$ & $90 \mathrm{mEq}$ & $40 \mathrm{mEq}$ & - & - \\
\hline Labetalol & $100 \mathrm{mg}$ & $200 \mathrm{mg}$ & $200 \mathrm{mg}$ & $200 \mathrm{mg}$ & $100 \mathrm{mg}$ \\
\hline Thiamine & - & $100 \mathrm{mg}$ & $100 \mathrm{mg}$ & $100 \mathrm{mg}$ & $100 \mathrm{mg}$ \\
\hline Folate & - & $1 \mathrm{mg}$ & $1 \mathrm{mg}$ & $1 \mathrm{mg}$ & $1 \mathrm{mg}$ \\
\hline Lactulose & - & $30 \mathrm{~mL}$ & $60 \mathrm{~mL}$ & $60 \mathrm{~mL}$ & $30 \mathrm{~mL}$ \\
\hline Enoxaparin & - & - & $40 \mathrm{mg}$ & $40 \mathrm{mg}$ & - \\
\hline Ondansetron & - & - & - & $4 \mathrm{mg}$ & - \\
\hline Dexmedetomidine & - & $100 \mathrm{~mL}$ & $100 \mathrm{~mL}$ & - & - \\
\hline Chlordiazepoxide & - & - & $75 \mathrm{mg}$ & $125 \mathrm{mg}$ & $100 \mathrm{mg}$ \\
\hline Lorazepam & $2 \mathrm{mg}$ & $5 \mathrm{mg}(\mathrm{PO})$ & $1 \mathrm{mg}(\mathrm{PO})$ & $25 \mathrm{mg}(\mathrm{PO})$ & - \\
\hline
\end{tabular}

This patient presented in stable condition at the psychiatric hospital but began showing signs of impending DTs within 24 hours of admission. Through continuous monitoring and cognitive testing, the team was able to identify progressive withdrawal and transfer him to intensive medical care before his condition fully deteriorated, possibly preventing the onset of seizures, autonomic instability, and death. He required constant benzodiazepine and frequent dexmedetomidine dosing to prevent full exacerbation of symptoms and needed continuous monitoring for agitation, confusion, and fluctuating cognition. Without the transfer, his condition would have become unmanageable on the psychiatric unit.

Table 3. Clinical and Laboratory Biomarkers Predicting Acute Alcohol Withdrawal in Index Patient

\begin{tabular}{|c|c|}
\hline Biomarker & Index Patient \\
\hline Thrombocytopenia & $\begin{array}{l}\text { Persisted through length of } \\
\text { admission }\end{array}$ \\
\hline Hypokalemia & Resolved HD4 \\
\hline Hyperhomocysteinemia & Resolved HD4 \\
\hline Hypertension & $\begin{array}{l}\text { Persisted through length of } \\
\text { admission }\end{array}$ \\
\hline Hypomagnesemia & Resolved HD4 \\
\hline Tachycardia & $\begin{array}{l}\text { Persisted through length of } \\
\text { admission }\end{array}$ \\
\hline
\end{tabular}

Treatment of DTs requires appropriate equipment, and studies have clearly shown that critically ill patients with frank signs of DTs are best managed in an inpatient medical units. ${ }^{6}$ Experts agree that IV benzodiazepines are critical for acute control of symptoms of alcohol withdrawal-like seizures. ${ }^{5}$ Nutritional support, especially in prevention of Wernicke's encephalopathy, often requires IV, not oral, administration of thiamine. ${ }^{5}$ This, coupled with other electrolyte imbalances, such as hypokalemia and hypomagnesemia, creates a complicated roadblock to recovery. If patients at risk for DTs are missed, they often end up on psychiatric units less equipped to handle treatment. Many inpatient psychiatry units have strict policies against IV lines and other tubes, including Foley catheters, preventing necessary treatment, leading to worse outcomes. In this case, this was only avoided by monitoring and prompt transfer. Thus, proper screening of at-risk patients in the ED is essential for proper identification and management.

In institutes with CIWA-Ar protocols, disposition is often simpler for emergency physicians with a goal to discharge patients with CIWA-Ar scores less than 8 for outpatient detoxification. ${ }^{7}$ In centers lacking resources for such protocols, clinician judgement and low threshold for inpatient medical admission are necessary for early identification of DTs and disposition. Thus, it is critical that early predictors of alcohol withdrawal seizures and DTs are identified and 
monitored when possible. Several biochemical markers have received recent attention as early predictors of worse outcomes. Thrombocytopenia, hypokalemia, and hyperhomocysteinemia have been shown to be associated with the development of DTs. ${ }^{8-11}$

In study by Kim and Kim, $35.1 \%$ of patients with a low platelet count developed delirium tremens from a sample size of 97 patients. ${ }^{8}$ Low platelet count and high blood level of homocysteine were independent risk factors with high diagnostic sensitivity and specificity for the development of DT. In the study by Berggren, Fahlke and Berglund, 3\% of the patient developed delirium tremens out of a total sample of 334 patients. ${ }^{9}$ Among those with DT, a higher proportion had thrombocytopenia. The sensitivity and specificity of thrombocytopenia for the development of DT during the treatment period was $70 \%$ and $69 \%$, respectively. The positive predictive value (PPV) was $6 \%$ and the negative predictive value (NPV) was $99 \% .^{9}$

Two out of these three important clinical markers and predictors of delirium tremens (thrombocytopenia, hypokalemia) were present in this patient. Direct alcohol toxicity to the bone marrow has been proposed as the cause of thrombocytopenia with early intervention and reversal possibly preventing future development of seizures and death. ${ }^{8,10}$ Simple measures, such as administration of vitamin B12, folate, and pyridoxine to lower blood homocysteine levels, can also be used in management. Among alcohol-related problems, hypertension is common but under-evaluated in the emergency setting. ${ }^{12}$ The vasodilator effect of alcohol is often suppressed by sympathetic reactions during withdrawal. The hypothalamic-pituitary-adrenal system may be activated, producing excess catecholamines and cortisol, causing severe BP fluctuations, especially in chronic alcoholics. Our patient, who had chronic hypertension, had subtle but significant BP changes with increasing pressures during impending DTs. As such, monitoring BP during management seems essential.

\section{Conclusion}

Due to widespread alcohol use and abuse, alcohol withdrawal syndromes are fairly common. ${ }^{13}$ The CIWA-Ar protocol is being increasingly used with early initiation of benzodiazepine therapy for successful management of these syndromes. However, in hospitals and medical centers with limited resources, nursing staff and psychiatric units need an alternate strategy to assist in early identification and treatment in the ED itself prior to transfer becomes imperative. Monitoring biochemical markers, such as platelet counts and electrolytes, and BP may be the answer.

\section{ACKNOWLEDGEMENT}

I would like to acknowledge the Department of Psychiatry at Texas Tech University Health Sciences Center and Professor Marina Chavez for her continued support in drafting this manuscript.

Article citation: Sarangi A. Alcohol withdrawal. The Southwest Respiratory and Critical Care Chronicles 2020;8(35):55-60

From: Department of Psychiatry, Texas Tech University Health Sciences Center, Lubbock, Texas

Submitted: $5 / 27 / 2020$

Accepted: $7 / 5 / 2020$

Reviewer: Drew Payne DO

Conflicts of interest: none

This work is licensed under a Creative Commons Attribution-ShareAlike 4.0 International License.

\section{REFERENCES}

1. Schuckit MA. Recognition and management of withdrawal delirium (delirium tremens). N Engl J Med 2014;371: 2109-2113.

2. Mainerova B, Prasko J, Latalova K, et al. Alcohol withdrawal delirium-diagnosis, course and treatment. Biomed Pap Med Fac Univ Palacky Olomouc Czech Repub 2015;159:44-52.

3. Maldonado JR, Sher Y, Ashouri JF, et al. The Prediction of Alcohol Withdrawal Severity Scale (PAWSS): systematic literature review and pilot study of a new scale for the prediction of complicated alcohol withdrawal syndrome. Alcohol 2014;48:375-390.

4. Sullivan JT, Sykora K, Schneiderman J, et al. Assessment of alcohol withdrawal: the revised clinical institute withdrawal assessment for alcohol scale (CIWA-Ar). Br J Addict 1989;84:1353-1357. 
5. Long D, Long B, Koyfman A. The emergency medicine management of severe alcohol withdrawal. Am J Emerg Med 2017;35:1005-1011.

6. Sieber E, Binting S, Willich SN. [Inpatient withdrawal treatment of patients suffering from alcohol addiction in Berlin (19931999)]. Gesundheitswesen (Bundesverband der Arzte des Offentlichen Gesundheitsdienstes (Germany)). 2003;65:81-89.

7. Maldonado JR. Novel algorithms for the prophylaxis and management of alcohol withdrawal syndromes-beyond benzodiazepines. Crit Care Clin 2017;33:559-599.

8. Kim DW, Kim HK, Bae EK, et al. Clinical predictors for delirium tremens in patients with alcohol withdrawal seizures. Am J Emerg Med 2015;33:701-704.

9. Berggren U, Fahlke C, Berglund KJ, et al. Thrombocytopenia in early alcohol withdrawal is associated with development of delirium tremens or seizures. Alcohol and Alcoholism 2009; 44:382-386.

10. Latvala J, Parkkila S, Niemela O. Excess alcohol consumption is common in patients with cytopenia: studies in blood and bone marrow cells. Alcohol Clin Exp Res 2004; 28:619-624.

11. Eyer F, Schuster T, Felgenhauer N, et al. Risk assessment of moderate to severe alcohol withdrawal-predictors for seizures and delirium tremens in the course of withdrawal. Alcohol Alcohol 2011;46:427-433.

12. Ceccanti M, Sasso GF, Nocente $R$, et al. Hypertension in early alcohol withdrawal in chronic alcoholics. Alcohol Alcohol. 2006;41:5-10.

13. Carlson RW, Kumar NN, Wong-Mckinstry E, et al. Alcohol withdrawal syndrome. Crit Care Clin 2012;28:549-585. 